\title{
Creación de un cuestionario de clima organizacional para hospitales de alta complejidad, Chile*
}

\section{Creation of a Questionnaire of Organizational Climate for Hospitals of High Complexity, Chile}

\section{Criação de um questionário de clima organizacional para hospitais de alta complexidade, Chile}

Fecha de recepción: 15-01-16 Fecha de aceptación: 15-05-16 Disponible en línea: 03-05-16 doi:10.11144/Javeriana.rgyps15-30.ccco

Cómo citar este artículo:

Bustamante-Ubilla MA, Lapo-Maza MC, Grandón Avendaño ML. Creación de un cuestionario de clima organizacional para hospitales de alta complejidad, Chile. Rev. Gerenc. Polít. Salud. 2016; 15(30): 126-141. http://dx.doi.org/10.11144/Javeriana.rgyps15-30.ccco

Miguel Alejandro Bustamante-Ubilla**

María del Carmen Lapo-Maza***

Maderline Lissette Grandón-Avendaño****

Artículo de investigación; proyecto: "Análisis consolidado del clima organizacional de dos hospitales de la región del Maule"; fecha de inicio: septiembre del 2013; fecha término: diciembre del 2013; sin financiamiento.

** Autor principal. Doctor en Ciencias Económicas y Empresariales, profesor asociado, Escuela de Ingeniería Comercial, Facultad de Economía y Negocios, Universidad de Talca, Campus Lircay, Avenida Lircay S/N, Talca - Chile. Correo electrónico:mabu@utalca.cl

**** Directora del Máster en Gerencia en Servicios de la Salud y Administración de Empresas, docente titular principal, Facultad de Ciencias Económicas y Administrativas, Universidad Católica de Santiago de Guayaquil, Guayaquil, Ecuador. Correo electrónico: maria.lapo@cu.ucsg.edu.ec

**** Ingeniero comercial, mención Administración. Alumna del Magíster en Gestión de Sistemas de Salud, Universidad de Talca. Asistente de investigación, Centro de Gestión de Instituciones de Salud, Facultad de Economía y Negocios, Universidad de Talca Campus Lircay. Correo electrónico: mgrandon@utalca.cl 


\section{Resumen}

En el contexto de un sector salud chileno en proceso de transformaciones, se propuso crear un cuestionario validado para medir el clima organizacional en hospitales de alta complejidad. Para su formulación se identificaron dimensiones y variables de diversos autores y se revisaron diferentes cuestionarios, validaciones y constructos utilizados en diferentes propuestas a nivel internacional. La investigación es de tipo descriptivo, cuantitativo e inferencial y se realizó sobre una base de datos de 561 empleados hospitalarios, a quienes se les aplicó un cuestionario por la vía de una entrevista personalizada en el lugar de trabajo, que consta de 71 variables agrupadas en 14 dimensiones. La aplicación del instrumento, así como los estudios de validez y de confiabilidad, permitieron dar origen a un nuevo cuestionario validado que cuenta con 10 factores y 44 reactivos de alta confiabilidad, que explican el $52,181 \%$ de la varianza total, alcanzando un alfa de Cronbach total de 0,89.

Palabras clave: clima organizacional; ciencias de la conducta; disciplinas y actividades conductuales; investigación conductal; psicología social; entrevista psicológica

\section{Abstract}

In the context of a Chilean health sector undergoing a process of transformations, it was proposed to create a validated questionnaire to measure the organizational climate in high complexity hospitals. In order to prepare it, dimensions and variables by diverse authors were identified and diverse questionnaires, validations and constructs used in different proposals at the international level were reviewed. The research is descriptive, quantitative and inferential and it was conducted on a database of 561 hospital employees, to whom a questionnaire was applied, through a personalized interview at their work place, consisting of 71 variables grouped in 14 dimensions. The application of the instrument, as well as the validity and reliability studies, allowed giving origin to a new validated questionnaire with 10 factors and 44 high-reliability reagents that explain $52.181 \%$ of the total variance, reaching a total Cronbach's alpha 0.89 .

Keywords: organizational climate; behavioral sciences; behavioral disciplines and activities; behavioral research; social psychology; psychological interview

\section{Resumo}

No contexto de um processo de transformações do setor saúde chileno, propõe-se criar um questionário validado para medir o clima organizacional em hospitais de alta complexidade. Para sua formulação dimensões e variáveis de diversos autores foram identificadas e se revisaram diferentes questionários, validações e construtos utilizados em diferentes propostas do nível internacional. A pesquisa é de tipo descritivo, quantitativo e inferencial e foi realizado sobre uma base de dados de 561 funcionários hospitalares, a quem foi aplicado questionário pela via de entrevista pessoalizada no local de trabalho, que consta de 71 variáveis agrupadas em 14 dimensões. A aplicação do instrumento, bem como os estudos de validez e confiabilidade, permitiu dar origem a um novo questionário validado que conta com 10 fatores e 44 reagente de alta confiabilidade, que explicam o 52,181 \% da variância total, atingindo um alfa de Cronbach total de 0,89 .

Palavras-chave: clima organizacional; ciências da conduta; disciplinas e atividades condutuais; pesquisa condutual; psicologia social; entrevista psicológica 


\section{Clima organizacional}

El clima organizacional ha sido definido y estructurado como un constructo complejo y diverso (tabla 1). A modo de ejemplo, se define como "el conjunto de propiedades medibles del ambiente de trabajo que directa o indirectamente influyen en la conducta de las personas" $(1,2)$, o como "el conjunto de características permanentes que describen una organización, la distinguen de otra e influye en el comportamiento de los individuos", y se mide por medio de percepciones $(3,4)$, en particular mediante la escala Likert como medio instrumental pertinente y validado (1).

Diversas propuestas para comprender el clima organizacional cuantifican una multiplicidad de dimensiones, algunas contextualizan la realidad (4), otras definen un patrón de interacciones de las personas en la organización (5), en tanto que también se caracterizan prácticas en un sistema y en un ambiente determinado (2), además de identificar elementos intervinientes que contextualizan la realidad organizacional $(6,7)$, contribuyendo desde diversas perspectivas a profundizar los estudios de clima organizacional.

Sin embargo, en estudios del ámbito sectorial de la salud se ha llegado a determinar que directivos y funcionarios coinciden en validar el saber y la experticia de los profesionales como elementos clave para lograr el éxito personal e institucional (8) y, sobre esa base, generar climas organizacionales adecuados al propósito institucional de prestar, con responsabilidad social, servicios de salud a la comunidad (4), llegándose a identificar hasta ochenta ítems influyentes en la conducta de un trabajador (9).

A nivel teórico-práctico, se ha relacionado el clima organizacional con la calidad de los servicios de salud, lo que se ha ratificado mediante 83 investigaciones de enfoque multidimensional (10), pero sin perjuicio de la certeza de las relaciones establecidas. Para su eficaz y pertinente aplicación, deben ser validadas en lo teórico, contextos y realidades específicas. Consecuentemente, desde una perspectiva más bien empírica y sistémica, a nivel de los servicios de salud pública, se ha llegado a determinar que el clima organizacional es esencial en los procesos de gestión, cambio e innovación, en consideración del rol técnicocientífico que el clima tiene sobre los procesos sociales que involucra, por una parte, porque el mismo clima organizacional es una herramienta estratégica diagnóstica vital y, por otra, porque algunos estudios han confirmado que las percepciones que contienen las mediciones de clima son esenciales para el desarrollo de las instituciones de salud pública (11).

Por otra parte, asumiendo la perspectiva multidimensional mencionada, estudios realizados a nivel internacional han analizado el impacto del clima organizacional en la seguridad de los individuos (12) y en la salud pública (13), confirmando la existencia de un panorama de estudios de clima de la mayor relevancia (14). En principio, se ha estudiado la multidimensionalidad de los factores de motivación hospitalaria (15), desempeño de los empleados, productividad, satisfacción, compromiso y calidad de trabajo $(16,17)$ $\mathrm{y}$, seguidamente, se ha logrado determinar que la mejor gestión de estos genera entornos laborales favorables $(18,19)$ que son percibidos con claridad por los médicos de familia (20) y que progresivamente propician estudios focalizados a nivel de unidades específicas de nivel hospitalario $(21,22)$ y, por esa vía, se busca mejorar los resultados y la calidad de atención a los usuarios de las comunidades específicas a las cuales estas entidades de salud sirven (23-25).

En suma, el clima organizacional es efectivamente un constructo multidimensional que incluye una diversidad de características complementarias que conforman una totalidad compleja. (26) Esta, de ser bien gestionada, es capaz de generar ambientes laborales mo- 
tivacionales positivos (27) que posibilitan la satisfacción de los empleadosy, a través de ello, la prestación de servicios de salud de calidad a la comunidad de usuarios que atiende $(24,28)$.

En la tabla 1, que sintetiza las dimensiones y variables que diversos autores identifican como constitutivas del clima organizacional, se observa que las quince aportaciones contienen entre dos y once variables, totalizando 105 variables o dimensiones que en conjunto definen las diversas visiones conceptuales que dichos autores sostienen.
En general, las variables propuestas muestran coincidencias relevantes que facilitan la conformación de agregados, los cuales en este trabajo se denominan dimensiones constitutivas del clima organizacional. Además de los estudios realizados por Cardona y Zambrano (41) a lo largo de quince años, se identificaron diez trabajos que utilizan hasta veinte dimensiones y, de ellas, ocho como las más relevantes, entre las cuales mencionan toma de decisiones, claridad organizacional, liderazgo, interacción social, motivación institucional, sistema de recompensas e incentivos,

Tabla 1. Dimensiones del Clima organizacional

\begin{tabular}{|c|c|c|}
\hline $\begin{array}{l}\text { Gavin, } 1975(\mathbf{2 9}) \\
\text { Estructura organizacional } \\
\text { Obstáculo } \\
\text { Recompensa } \\
\text { Espíritu de trabajo } \\
\text { Confianza y consideración de } \\
\text { Parte de los administradores } \\
\text { Riesgos y desafíos }\end{array}$ & $\begin{array}{l}\text { Campbell et al., } 1970(\mathbf{3 0}) \\
\text { Autonomía individual } \\
\text { Estructura } \\
\text { 3. Orientación de la } \\
\text { recompensa } \\
\text { 4. Consideración } \\
\text { 5. Calidez } \\
\text { 6. Apoyo }\end{array}$ & $\begin{array}{l}\text { Jones y James, } 1979(\mathbf{3 1}) \\
\text { Categoría: Características de } \\
\text { trabajo y rol } \\
\text { Categoría: Característica de } \\
\text { liderazgo } \\
\text { Categoría: Característica de } \\
\text { grupo de trabajo } \\
\text { Categoría: Característica de la } \\
\text { organización }\end{array}$ \\
\hline $\begin{array}{l}\text { Pritchard y Karasick, } 1973 \text { (32) } \\
\text { Autonomía } \\
\text { Conflicto contra cooperación } \\
\text { Relaciones sociales } \\
\text { Estructura organizacional } \\
\text { Recompensa } \\
\text { Relación entre rendimiento y } \\
\text { remuneración } \\
\text { Niveles de ambición de la } \\
\text { empresa } \\
\text { Estatus } \\
\text { Flexibilidad e innovación } \\
\text { Centralización } \\
\text { Apoyo }\end{array}$ & $\begin{array}{l}\text { Newman, } 1977(\mathbf{3 3}) \\
\text { Estilo de supervisión } \\
\text { Característica de la tarea } \\
\text { Relación desempeño- } \\
\text { recompensa } \\
\text { Motivación laboral } \\
\text { Equipo y distribución de } \\
\text { personas y materiales } \\
\text { Entrenamiento } \\
\text { Políticas sobre toma de } \\
\text { decisiones } \\
\text { Espacio de trabajo } \\
\text { Presión para producir } \\
\text { Responsabilidad e importancia } \\
\text { del trabajo }\end{array}$ & $\begin{array}{l}\text { Steers, } 1977(\mathbf{3 4}) \\
\text { Estructura organizacional } \\
\text { Refuerzo } \\
\text { Centralización del poder } \\
\text { 4. Posibilidad de cumplimiento } \\
\text { Formación y desarrollo } \\
\text { Seguridad contra riesgo } \\
\text { Apertura contra rigidez } \\
\text { Estatus y moral } \\
\text { 9. Reconocimiento y } \\
\text { retroalimentación } \\
\text { 10. Competencia } \\
\text { 11. Flexibilidad organizacional }\end{array}$ \\
\hline $\begin{array}{l}\text { Likert, } 1976 \text { (1) } \\
\text { 1. Métodos de mando } \\
\text { 2. Naturaleza de las fuerzas de } \\
\text { motivación } \\
\text { 3. Naturaleza de los procesos de } \\
\text { comunicación } \\
\text { 4. Naturaleza de los procesos de } \\
\text { influencia y de interacción } \\
\text { 5. Toma de decisiones } \\
\text { 6. Fijación de los objetivos y de } \\
\text { las directrices } \\
\text { 7. Proceso de control } \\
\text { 8. Objetivos de resultados y de } \\
\text { perfeccionamiento }\end{array}$ & $\begin{array}{l}\text { Payne et al., } 1971 \text { (35) } \\
\text { Tipo de organización } \\
\text { Control }\end{array}$ & $\begin{array}{l}\text { Lawler } \text { et al., } 1974(\mathbf{3 6}) \\
\text { 1. Competencia, eficacia } \\
\text { 2. Responsabilidad } \\
\text { 3. Nivel práctico concreto } \\
\text { 4. Riesgo } \\
\text { 5. Impulsividad }\end{array}$ \\
\hline
\end{tabular}




\begin{tabular}{|c|c|c|}
\hline $\begin{array}{l}\text { Litwin y Stringer, } 1968(3) \\
\text { 1. Estructura } \\
\text { 2. Responsabilidad } \\
\text { 3. Recompensa } \\
\text { 4. Riesgo } \\
\text { 5. Calidez } \\
\text { 6. Apoyo } \\
\text { 7. Normas } \\
\text { 8. Conflicto } \\
\text { 9. Identidad }\end{array}$ & $\begin{array}{l}\text { Schneider y Bartlett, } 1968 \text { (37) } \\
\text { 1. Apoyo proveniente de la } \\
\text { dirección } \\
\text { 2. Interés por los nuevos } \\
\text { empleados } \\
\text { 3. Conflicto } \\
\text { 4. Independencia de los agentes } \\
\text { 5. Satisfacción } \\
\text { 6. Estructura organizacional }\end{array}$ & $\begin{array}{l}\text { Mever, } 1968(38) \\
\text { Conformidad } \\
\text { Responsabilidad } \\
\text { Normas } \\
\text { Recompensa } \\
\text { Claridad organizacional } \\
\text { Espíritu de trabajo }\end{array}$ \\
\hline $\begin{array}{l}\text { Halpin y Crofts, } 1963(2) \\
\text { Cohesión entre el cuerpo } \\
\text { docente } \\
\text { Grado de compromiso del } \\
\text { cuerpo docente } \\
\text { Moral de grupo } \\
\text { Apertura de espíritu } \\
\text { Consideración } \\
\text { Nivel afectivo de las relaciones } \\
\text { con la dirección } \\
\text { Importancia de la producción }\end{array}$ & $\begin{array}{l}\text { Forehand y Gilmer, } 1964 \text { (39) } \\
\text { 1. Tamaño de la organización } \\
\text { 2. Estructura organizacional } \\
\text { 3. Complejidad sistemática de } \\
\text { la organización } \\
\text { 4. Estilo de liderazgo } \\
\text { 5. Orientación de fines }\end{array}$ & $\begin{array}{l}\text { Friedlander y Margulies, } 1969 \\
\underline{(40)} \\
\text { Empeño } \\
\text { Obstáculos o trabas } \\
\text { Intimidad } \\
\text { Espíritu de trabajo } \\
\text { Actitud } \\
\text { 6. Acento puesto sobre la } \\
\text { producción } \\
\text { Confianza } \\
\text { Consideración }\end{array}$ \\
\hline
\end{tabular}

Fuente: elaboración propia sobre la base de los autores mencionados

apertura organizacional y supervisión, y que a causa de las realidades en que fueron validados, ninguno de los trabajos aplica en su totalidad. De hecho, solo uno de ellos aplica siete de las dimensiones mencionadas (42), y tres trabajos, tales como IPAO, OCM y BSC, aplican solo seis dimensiones $(43,44,5)$.

En síntesis y a modo de corolario, el estudio de los climas en organizaciones de salud es claramente necesario y relevante, por el impacto en los empleados y los efectos que tiene sobre los usuarios que reciben atención, razón por la cual este trabajo propone un cuestionario multidimensional debidamente validado para la medición del clima organizacional en hospitales de alta complejidad.

\section{Material y métodos}

Se realizó un estudio descriptivo, transversal y retrospectivo, con un diseño de investigación exploratorio e inferencial $(45,46)$, que recoge información cuali-cuantitativa de percepción de los componentes del clima organizacional. Se utilizó el criterio de inclusión para la deter- minación de una muestra estratificada de 561 elementos, considerando a los empleados del sector salud provenientes de dos hospitales complejos de la Región del Maule de Chile.

El trabajo de campo se realizó en el año 2013, con la aplicación de un cuestionario cuyos reactivos son medidos a través de una escala Likert creciente de cinco puntos (47), donde el valor 1 indica estar totalmente en desacuerdo con la afirmación y el valor 5 señala estar totalmente de acuerdo.

Los datos fueron procesados mediante el software estadístico sPss versión 15.0 e incluyó el análisis descriptivo, la definición demográfica de la muestra (46) y un análisis inferencial a través de factores de componentes principales (48).

\section{Descripción de los procedimientos utilizados}

Mediante sesiones de grupo foco se identificaron situaciones y contextos laborales asociados a dimensiones y variables relacionadas con el clima organizacional (49), recogiéndose 
percepciones en los términos y palabras expresados por los actuantes para evitar interpretaciones erradas (31). Las expresiones finalmente seleccionadas fueron validadas y confirmadas por los propios participantes.

Sobre la base de la identificación de reactivos obtenidos en la revisión teórica (tabla 1), y de las percepciones recogidas en los talleres de grupo focal, se estructuró un cuestionario genérico que dio origen a una primera versión del instrumento, estructurado en 14 dimensiones que contienen 71 reactivos, que fueron debidamente ratificados mediante sesiones de trabajo colectivo con una muestra de funcionarios de salud, utilizando el método zopp $(8,49)$, y por medio de una consulta a directivos de salud y académicos expertos (50) que permitió validar las variables y dimensiones provenientes de la teoría (tabla 1) con la síntesis de reactivos sintetizados del grupo focal.

\section{Elaboración del cuestionario de clima orga- nizacional}

Se determinaron catorce dimensiones de síntesis, a saber: estructura, responsabilidad, recompensa, riesgo, calidez, apoyo, administración del conflicto, identidad, estilo de supervisión, motivación laboral, estabilidad laboral, oportunidad de desarrollo, comunicación, y equipos y distribución de personas $y$ material, que contienen 71 afirmaciones o negaciones (tabla 1 ) definidas como reactivos para el cuestionario.

El cuestionario se estructuró en dos partes. La primera contiene los reactivos que los entrevistados deben responder, utilizando una escala Likert de cinco puntos con intervalos $(1,47,51)$, donde destaca el hecho de que las preguntas número $2,3,4,7,8,11,15,19,21$, 22, 23, 26, 28 a 30, 40, 42, 48, 53, 58 a 60, 62, 63,64 y 67 son negaciones y se analizan con una escala invertida en la que el número 3 de la escala tiene valor neutro. La segunda parte se organizó para recoger los datos demográficos de la muestra para su debida caracterización.

\section{Piloteo y forma de aplicación del instrumento}

El pre-testeo del cuestionario para chequear comprensión, lenguaje, formato, instrucciones y escalas de respuesta, y descartar dudas respecto de las preguntas, se realizó con una muestra aleatoria de treinta sujetos (49) que incluyó los niveles directivo y funcionarios de planta de la segunda línea de mando, lo que definió el criterio de exclusión, de modo que no fuesen incorporados en la muestra del estudio general $(51,52)$. La validación por expertos se realizó con nueve académicos, para ratificar lenguaje, unidireccionalidad, estructura semántica, consistencia y validez de las taxonomías teóricas utilizadas (46).

Realizados estos procedimientos se estructuró el cuestionario, que en este trabajo se denomina Cuestionario de Clima Organizacional Original (CCOO), y que fue el aplicado en el estudio general de validación del instrumento, dando origen al Cuestionario de Clima Organizacional Validado (CCOV).

El trabajo de campo se realizó en tres semanas de octubre del 2013 y abordó las unidades clínicas y administrativas en forma personalizada, aplicando el $\mathrm{CCOO}$ en forma anónima (51), ocupando un lapso de $45 \mathrm{mi}$ nutos en cada caso, y se ejecutó conforme a las leyes chilenas $\mathrm{n}^{\mathrm{o}} 19.628$ (53) y no 17.374 (54) de reserva de la información personal del entrevistado.

\section{Validez de contenido, constructo y convergente}

Se verificó la validez de contenido, sobre la base de diversas teorías (tabla 1): la de constructo a través de grupos focales, definición 
operativa de las variables y consulta a expertos (50); la validez convergente, mediante el análisis de factores de componentes principales, con previa verificación de pertinencia del índice кмо у prueba de esfericidad de Bartlett (tabla 2), lográndose un índice кMо cercano a 0,90 y esfericidad $(p)$ significativa. Finalmente, la validez discriminante se confirmó por las varianzas explicadas de los factores y las cargas de los componentes con cada factor, superiores o iguales a $0,5(46,48,49)$.

\section{Análisis de confiabilidad}

Para ratificar la consistencia de la presente propuesta de cuestionario de clima organizacional, previamente se analizaron diversos estudios que proponen instrumentos similares, y se encontró desde ausencia de datos como es el caso del ICO (42) — hasta otras que presentan los medios de prueba pertinentes para dichos estudios, tales como el IPAO (43), C-CLIOU (55) y CFK Ltd. (56), quienes aplicaron alfa de Cronbach y alcanzaron un índice muy alto de 0,90. En otros estudios, sin embargo, que si bien lograron coeficientes elevados, algunas de sus dimensiones no alcanzaron coeficientes suficientes, como es el caso de MDCo (57), que de acuerdo con el criterio científico sería inaceptable (48).

Sobre la base de lo indicado y valorando aciertos de estudios previos, se decidió la aplicación de tres niveles de análisis. Primero, se verificó la pertinencia de los datos para asegurar que los análisis estadísticos fuesen los adecuados (KMо y prueba de Bartlett); segundo, se analizó la confiabilidad a partir del coeficiente alfa de Cronbach del instrumento en su totalidad; y tercero, se ratificó la confiabilidad de cada una de las dimensiones incorporadas en el estudio, en este caso catorce dimensiones que contienen 71 reactivos, considerando el rango mínimo de 0,5 como criterio de confiabilidad mínima aceptable (49).

\section{Resultados}

\section{Contexto del estudio}

El cuestionario que se propone para la medición del clima organizacional en hospitales de alta complejidad de Chile, definidos como tales mediante la Norma Técnica $n^{\circ}$ 0150, Resolución Exenta ${ }^{\circ} 646$ del Ministerio de Salud de Chile (58), fue validado con una muestra de 561 empleados de dos hospitales chilenos.

El primero de los hospitales se ubica en una capital provincial con una población de 266952 habitantes, un alcance geográfico de atención de 156675 personas y una cobertura del $92,8 \%$ de la población asignada. Se cuenta con 252 camas, 18 cunas y un total de 835 funcionarios. El segundo es de carácter regional e incluye cuatro provincias cuya población aproximada es de un millón de personas; su rango de atención efectiva es de 353019 personas. Cuenta con una plantilla de 1468 funcionarios para 577 camas que atiende a un total de 280114 beneficiarios de la capital regional (59).

\section{Análisis descriptivo}

De la muestra de 561 sujetos, un $62 \%$ corresponde a mujeres, 26,92 y $23,71 \%$ de las cuales se ubican en los rangos 26 a 35 y 46 a 55 años, respectivamente. Un $49 \%$ cuenta con educación superior completa y un $21 \%$ registra educación media completa. Los años de servicio se concentran en los extremos: un $36 \%$ registra más de 21 años y un $25 \%$ entre 1 y 5 años de servicio.

\section{Resultados del análisis de confiabilidad}

Sobre la base del análisis de confiabilidad (60) de los factores de componentes principales, realizado mediante matriz de componentes rotados, para los cuestiona- 
rios Original y Validado (48) (tabla 2), el resultado obtenido permite afirmar que los instrumentos alcanzan un índice de 0,89 (60), lo cual los ubica en un rango de muy alta confiabilidad $(61,49)$.

Se observa que el cCoo (tabla 2) totaliza las 14 dimensiones y 71 variables iniciales del estudio. Se verifica que la dimensión Motivación laboral se ubica en el borde del rango muy alta, de acuerdo con la clasificación de Ruiz (49). En el rango alta se ubican las dimensiones equiposy distribución de personasy material, calidez, estabilidad laboral y apoyo y comunicación. En tanto que las dimensiones riesgo, estructura, oportunidad de desarrollo, recompensa, identidad y estilo de supervisión alcanzan la magnitud moderada. Finalmente, las dimensiones administración del conflicto y responsabilidad logran magnitudes bajas.

Por otra parte, el ccov (tabla 2), presenta 11 factores y 46 reactivos; sin embargo, por alcanzar un alfa de Cronbach bajo, de 0,389, considerado insuficiente para el estudio, se elimina el "Factor 11 Excluido", junto a sus dos componentes. En consecuencia, se confirman diez factores, a saber: motivación laboral, apoyo al emprendimiento; organización y desempeño; colaboración y buen trato; equipos y distribución de personas y material; autonomía y profesionalismo; desarrollo profesional; estabilidad laboral e iniciativa participativa, que alcanzan confiabilidad alta, además del factor comunicación y desarrollo que, aun cuando logra solo confiabilidad moderada, permite totalizar 44 componentes principales que explican el 52,181\% de la varianza total y alcanza una confiabilidad total del instrumento de $0,89 \%$ (tabla 2).

\section{Análisis de factores de componentes prin- cipales del ccov}

El primer factor (tabla 3), esto es, colaboración y buen trato, se compone de las dimensiones apoyo y estilo de supervisión, destacando que la colaboración entre pares y la ayuda, apoyo y trato agradable de los altos mandos genera un clima organizacional que influye positivamente en la motivación y en el comportamiento de los funcionarios. En este mismo sentido, el segundo factor, motivación laboral, subraya el compromiso de los trabajadores por realizar un trabajo eficiente $\mathrm{y}$, por lo mismo, transforma esta variable en un pilar fundamental del clima organizacional. De manera que, en conjunto, estos dos primeros factores definen lo que aquí se llamará personalidad de las organizaciones, por cuanto son los componentes relacionales, motivacionales y de integración resultantes de las percepciones de las personas los que la definen y generan las bases para su desarrollo (15).

El tercer factor, iniciativa participativa, releva la importancia de la inclusión de las personas en el trabajo cotidiano y destaca cómo las nuevas ideas contribuyen al buen desempeño en tanto encuentren el respaldo de los superiores. En directa relación, el cuarto factor, desarrollo profesional, muestra cómo los sistemas de ascensos y promociones otorgan oportunidades, dando espacios para la concreción de habilidades y talentos que se expresan en la asignación de responsabilidades incrementales y de confianza que los funcionarios valoran. Por una parte, se otorgan espacios de autonomía e independencia progresivamente logrados y, por otra, se valoran las especializaciones que les han permitido alcanzar éxitos en sus respectivos estamentos profesionales. En esta misma línea de análisis, el quinto factor, apoyo al emprendimiento, promueve y estimula la generación de nuevas ideas como componente clave del desempeño que los funcionarios aprecian al contar con apoyo directivo y al percatarse de que se les recibe con respeto y confianza (4). Asociado a este factor, los funcionarios reconocen este elemento como una 
Tabla 2. Alfa de Cronbach Cuestionarios Original y Validado, 2013

\begin{tabular}{|c|c|c|c|c|c|}
\hline \multicolumn{3}{|c|}{ Cuestionario Original ( $\left.\mathrm{ccoo}^{*}\right)$} & \multicolumn{3}{|c|}{ Cuestionario Validado (ccov**) } \\
\hline Dimensiones & $\begin{array}{c}\delta \\
\text { Cronbach }\end{array}$ & Variables & Factores & $\begin{array}{c}\delta \\
\text { Cronbach }\end{array}$ & Variables \\
\hline Estructura & 0,54 & 7 & $\begin{array}{l}\text { 1. Colaboración y buen } \\
\text { trato }\end{array}$ & 0,767 & 7 \\
\hline Responsabilidad & 0,27 & 8 & 2. Motivación laboral & 0,802 & 3 \\
\hline Recompensa & 0,49 & 6 & $\begin{array}{l}\text { 3. Iniciativa } \\
\text { participativa }\end{array}$ & 0,629 & 6 \\
\hline Riesgo & 0,57 & 4 & $\begin{array}{l}\text { 4. Desarrollo } \\
\text { profesional }\end{array}$ & 0,695 & 5 \\
\hline Calidez & 0,74 & 6 & $\begin{array}{l}\text { 5. Apoyo al } \\
\text { emprendimiento }\end{array}$ & 0,788 & 6 \\
\hline Apoyo & 0,68 & 5 & $\begin{array}{l}\text { 6. Equipos y } \\
\text { distribución de } \\
\text { personas y material }\end{array}$ & 0,732 & 3 \\
\hline $\begin{array}{l}\text { Administración del } \\
\text { conflicto }\end{array}$ & 0,39 & 5 & $\begin{array}{l}\text { 7. Organización y } \\
\text { desempeño }\end{array}$ & 0,772 & 4 \\
\hline Identidad & 0,56 & 7 & 8. Estabilidad laboral & 0,659 & 3 \\
\hline Estilo de supervisión & 0,43 & 6 & $\begin{array}{l}\text { 9. Comunicación y } \\
\text { desarrollo }\end{array}$ & 0,596 & 4 \\
\hline Motivación laboral & 0,80 & 3 & $\begin{array}{l}\text { 10. Autonomía y } \\
\text { profesionalismo }\end{array}$ & 0,707 & 3 \\
\hline Estabilidad laboral & 0,77 & 3 & \multirow{2}{*}{\multicolumn{2}{|c|}{ SUBTOTAL }} & \multirow{2}{*}{44} \\
\hline Oportunidad desarrollo & 0,52 & 3 & & & \\
\hline Comunicación & 0,63 & 5 & Factor 11: Excluido & 0,389 & 2 \\
\hline $\begin{array}{l}\text { Equipos y distribución } \\
\text { de personas y material }\end{array}$ & 0,79 & 3 & \multicolumn{2}{|l|}{ TOTAL } & 46 \\
\hline Todas las variables & 0,890 & 71 & Todas las variables & 0,890 & 44 \\
\hline \multicolumn{3}{|l|}{ кмо у prueba de Bartlett } & \multicolumn{3}{|c|}{ кмо у prueba de Bartlett } \\
\hline \multicolumn{2}{|c|}{$\begin{array}{l}\text { Medida de adecuación muestral de } \\
\text { Kaiser-Meyer-Olkin }\end{array}$} & 0,866 & \multicolumn{2}{|c|}{$\begin{array}{l}\text { Medida de adecuación muestral de } \\
\text { Kaiser-Meyer-Olkin }\end{array}$} & 0,862 \\
\hline \multirow{2}{*}{$\begin{array}{l}\text { Prueba de esfericidad } \\
\text { de Bartlett }\end{array}$} & gl. & 1128 & \multirow{2}{*}{$\begin{array}{l}\text { Prueba de esfericidad } \\
\text { de Bartlett }\end{array}$} & gl. & 946 \\
\hline & Sig. & 0,000 & & Sig. & 0,000 \\
\hline
\end{tabular}

Elaboración propia a través de sPss 15.0

$\left({ }^{*}\right)=$ Cuestionario de Clima Organizacional Original (cCOo)

$(* *)=$ Cuestionario de Clima Organizacional Validado ( $\mathrm{CCOV})$

palanca de cambio, estímulo y promoción que deriva progresivamente en el desarrollo de capacidades de intra-emprendimiento que, si son bien aplicadas, pueden ayudar a resolver desafíos clave de la organización.
El sexto factor, equipos y distribución de personas y material, refiere a la productividad mutua que se establece entre los factores productivos y que se manifiesta en coherencia, eficacia y eficiencia de las prestaciones de 
salud, confirmando que si son estructurados de manera pertinente, son claves para el logro de resultados. Asimismo, el séptimo factor, organización y desempeño, muestra que la organización y planificación de las obligaciones, así como la división de tareas, funciones y responsabilidades, deben coexistir con un sistema de recompensas y estímulos adecuados para fomentar el buen desempeño.

Por otra parte, el octavo factor, estabilidad laboral, representa la valoración que los funcionarios hacen de la seguridad del empleo, su pertenencia a una unidad de trabajo, y que los empleados perciben que influye directamente en el compromiso individual y colectivo de la organización. Del mismo modo, en lo operativo, el noveno factor, comunicación y desarrollo, pone de manifiesto la importancia de los sistemas formales de comunicación entre personas, servicios y unidades para que trabajen de forma articulada y armónica (50). En lo concreto, los funcionarios esperan conocer cuál es su cometido, qué es lo que se espera de ellos y qué es lo que se debe saber para ejecutar bien sus tareas; por lo mismo, valoran la coordinación interpersonal con sus jefaturas y pares, en un proceso de comunicación que debe ser fluido y permanente desde y hacia la dirección para la ejecución responsable de sus tareas.

Finalmente, el décimo factor, autonomía y profesionalismo, pone de manifiesto el valor de la libertad, la valoración de la autonomía y la necesidad de los funcionarios de contar con espacio y atribuciones, por una parte, que les permitan ejercer con independencia sus talentos y, por otra, que se reconozca el valor de la experticia, el dominio contextual

TABLA 3. FACTORES DE COMPONENTES PRINCIPALES

\begin{tabular}{|c|c|c|c|c|c|c|c|}
\hline \multicolumn{3}{|c|}{ Factor 1: Colaboración y buen trato } & $\delta$ & \multicolumn{3}{|c|}{ Factor 2: Motivación laboral } & $\delta$ \\
\hline \multicolumn{3}{|c|}{$\begin{array}{l}\text { Cuando tengo que hacer un trabajo } \\
\text { difícil puedo contar con la ayuda de } \\
\text { mis compañeros o colegas }\end{array}$} & 0,664 & \multicolumn{3}{|c|}{$\begin{array}{l}\text { En esta institución la gente está com- } \\
\text { prometida con su trabajo }\end{array}$} & 0,832 \\
\hline \multicolumn{3}{|c|}{$\begin{array}{l}\text { Los jefes entregan información a su } \\
\text { personal respecto de su desempeño } \\
\text { laboral }\end{array}$} & 0,561 & & & & \\
\hline \multicolumn{3}{|c|}{$\begin{array}{l}\text { Los funcionarios saben lo que los } \\
\text { supervisores esperan de ellos }\end{array}$} & 0,555 & \multicolumn{3}{|c|}{$\begin{array}{l}\text { Las personas de esta institución se } \\
\text { esfuerzan bastante por desarrollar } \\
\text { eficientemente su labor }\end{array}$} & 0,817 \\
\hline \multicolumn{3}{|c|}{$\begin{array}{l}\text { Los jefes promueven las buenas rela- } \\
\text { ciones humanas entre las personas de } \\
\text { la institución }\end{array}$} & 0,540 & \multirow{4}{*}{\multicolumn{3}{|c|}{$\begin{array}{l}\text { Las personas en esta institución mues- } \\
\text { tran interés por el trabajo que realizan }\end{array}$}} & \multirow{4}{*}{0,723} \\
\hline \multicolumn{3}{|c|}{$\begin{array}{l}\text { Los jefes de esta institución tratan con } \\
\text { respeto a sus subordinados }\end{array}$} & 0,508 & & & & \\
\hline \multicolumn{3}{|c|}{$\begin{array}{l}\text { Cuando tengo que hacer un trabajo } \\
\text { difícil puedo contar con la ayuda de } \\
\text { mi(s) jefe(s) }\end{array}$} & 0,508 & & & & \\
\hline \multicolumn{3}{|c|}{$\begin{array}{l}\text { En esta institución se trabaja en } \\
\text { equipo }\end{array}$} & 0,447 & & & & \\
\hline $\begin{array}{l}\text { Varianza } \\
\text { explicada \% }\end{array}$ & 5,898 & $\begin{array}{l}\text { Alfa de } \\
\text { Cronbach }\end{array}$ & 0,767 & $\begin{array}{l}\text { Varianza } \\
\text { explicada\% }\end{array}$ & 5,763 & $\begin{array}{l}\text { Alfa de } \\
\text { Cronbach }\end{array}$ & 0,802 \\
\hline
\end{tabular}




\begin{tabular}{|c|c|c|c|c|c|c|c|}
\hline \multicolumn{3}{|c|}{ Factor 3. Iniciativa participativa } & $\delta$ & \multicolumn{3}{|c|}{ Factor 4. Desarrollo profesional } & $\delta$ \\
\hline \multicolumn{3}{|c|}{$\begin{array}{l}\text { Aquí se da facilidad para que cualquier } \\
\text { funcionario pueda presentar una idea } \\
\text { nueva }\end{array}$} & 0,603 & \multicolumn{3}{|c|}{$\begin{array}{l}\text { En esta institución existen posibilida- } \\
\text { des de ascenso }\end{array}$} & 0,670 \\
\hline \multicolumn{3}{|c|}{$\begin{array}{l}\text { Los jefes son comprensivos cuando } \\
\text { uno comete un error }\end{array}$} & 0,591 & \multicolumn{3}{|c|}{$\begin{array}{l}\text { En esta institución se dan las oportuni- } \\
\text { dades para desarrollar las destrezas y } \\
\text { habilidades personales }\end{array}$} & 0,573 \\
\hline \multicolumn{3}{|c|}{$\begin{array}{l}\text { Uno progresa en esta institución si } \\
\text { tiene iniciativa }\end{array}$} & 0,550 & \multicolumn{3}{|c|}{$\begin{array}{l}\text { Los trabajadores de esta institución } \\
\text { creen más en la información oficial que } \\
\text { en el rumor que surge sobre un cierto } \\
\text { hecho }\end{array}$} & 0,549 \\
\hline \multicolumn{3}{|c|}{$\begin{array}{l}\text { En esta institución, entre el personal } \\
\text { predomina un ambiente de amistad }\end{array}$} & 0,474 & \multicolumn{3}{|c|}{$\begin{array}{l}\text { Esta institución se caracteriza por un } \\
\text { buen ambiente de trabajo }\end{array}$} & 0,504 \\
\hline \multicolumn{3}{|c|}{$\begin{array}{l}\text { La dirección de esta institución se } \\
\text { preocupa por las personas, de cómo se } \\
\text { sienten y de sus problemas }\end{array}$} & 0,362 & \multicolumn{3}{|c|}{$\begin{array}{l}\text { En esta institución se nos mantiene } \\
\text { informados sobre materias que debe- } \\
\text { ríamos saber }\end{array}$} & 0,471 \\
\hline \multicolumn{3}{|c|}{$\begin{array}{l}\text { Los jefes piensan que las discrepancias } \\
\text { entre las distintas unidades y personas } \\
\text { pueden ser útiles para el mejoramiento } \\
\text { de la institución }\end{array}$} & 0,212 & & & & \\
\hline $\begin{array}{l}\text { Varianza } \\
\text { explicada } \%\end{array}$ & 5,737 & $\begin{array}{l}\text { Alfa de } \\
\text { Cronbach }\end{array}$ & 0,629 & $\begin{array}{l}\text { Varianza } \\
\text { explicada } \%\end{array}$ & 5,650 & $\begin{array}{l}\text { Alfa de } \\
\text { Cronbach }\end{array}$ & 0,695 \\
\hline \multicolumn{3}{|c|}{ Factor 5: Apoyo al emprendimiento } & $\delta$ & \multicolumn{3}{|c|}{$\begin{array}{l}\text { Factor 6: Equipos y distribución de } \\
\text { personas y material }\end{array}$} & $\delta$ \\
\hline \multicolumn{3}{|c|}{$\begin{array}{l}\text { El ambiente en esta institución es pro- } \\
\text { picio para desarrollar nuevas ideas }\end{array}$} & 0,724 & \multicolumn{3}{|c|}{$\begin{array}{l}\text { La distribución del personal y de mate- } \\
\text { rial en esta institución permite que se } \\
\text { realice un trabajo eficiente y efectivo }\end{array}$} & 0,852 \\
\hline \multicolumn{3}{|c|}{$\begin{array}{l}\text { Las nuevas ideas que aporta el perso- } \\
\text { nal son bien acogidas por los jefes }\end{array}$} & 0,674 & \multicolumn{4}{|c|}{ realice un trabajo enciente y erectivo } \\
\hline \multicolumn{3}{|c|}{$\begin{array}{l}\text { En esta institución existen pocas } \\
\text { críticas }\end{array}$} & 0,488 & \multicolumn{3}{|c|}{$\begin{array}{l}\text { En esta institución hay disponibili- } \\
\text { dad de materiales del tipo y cantidad } \\
\text { requeridos }\end{array}$} & 0,812 \\
\hline \multicolumn{3}{|c|}{$\begin{array}{l}\text { En esta institución existe un alto respe- } \\
\text { to por las personas }\end{array}$} & 0,469 & \multicolumn{3}{|c|}{$\begin{array}{l}\text { El número de personas que trabaja en } \\
\text { esta institución es apropiado para la } \\
\text { cantidad de trabajo que se realiza }\end{array}$} & 0,753 \\
\hline \multicolumn{3}{|c|}{$\begin{array}{l}\text { En esta institución la gente confía en } \\
\text { los demás }\end{array}$} & 0,422 & & & & \\
\hline \multicolumn{3}{|c|}{$\begin{array}{l}\text { Siempre trabajamos en forma organi- } \\
\text { zada y con planificación }\end{array}$} & 0,374 & & & & \\
\hline $\begin{array}{l}\text { Varianza } \\
\text { explicada } \%\end{array}$ & 5,512 & $\begin{array}{l}\text { Alfa de } \\
\text { Cronbach }\end{array}$ & 0,732 & $\begin{array}{l}\text { Varianza } \\
\text { explicada } \%\end{array}$ & 5,007 & $\begin{array}{l}\text { Alfa de } \\
\text { Cronbach }\end{array}$ & 0,788 \\
\hline
\end{tabular}




\begin{tabular}{|c|c|c|c|c|c|c|c|}
\hline \multicolumn{3}{|c|}{ Factor 7: Organización y desempeño } & $\delta$ & \multicolumn{3}{|c|}{ Factor 8: Estabilidad laboral } & $\delta$ \\
\hline \multicolumn{3}{|c|}{$\begin{array}{l}\text { En esta institución los trabajos están } \\
\text { bien asignados y organizados }\end{array}$} & 0,646 & \multicolumn{3}{|c|}{$\begin{array}{l}\text { No me preocupa que por una reorgani- } \\
\text { zación en la institución se vea afectada } \\
\text { la estabilidad de mi trabajo }\end{array}$} & 0,802 \\
\hline \multicolumn{3}{|c|}{$\begin{array}{l}\text { La capacidad es el criterio básico para } \\
\text { asignar tareas en esta institución }\end{array}$} & 0,638 & \multicolumn{3}{|c|}{$\begin{array}{l}\text { No me preocupan los efectos que pue- } \\
\text { dan tener sobre la estabilidad de mi em- } \\
\text { pleo los cambios de método de trabajo } \\
\text { y la automatización en esta institución }\end{array}$} & 0,793 \\
\hline \multicolumn{3}{|c|}{$\begin{array}{l}\text { En esta institución los que se desem- } \\
\text { peñan mejor en su trabajo obtienen un } \\
\text { mayor reconocimiento de parte de sus } \\
\text { superiores }\end{array}$} & 0,543 & \multirow{2}{*}{\multicolumn{3}{|c|}{$\begin{array}{l}\text { No creo que en cualquier momento pue- } \\
\text { da perder el trabajo en esta institución }\end{array}$}} & 0,737 \\
\hline $\begin{array}{l}\text { En esta instit } \\
\text { quién tiene q }\end{array}$ & $\begin{array}{l}\text { ción siem } \\
\text { e decidir }\end{array}$ & $\begin{array}{l}\text { re se sabe bien } \\
\text { as cosas }\end{array}$ & 0,464 & & & & \\
\hline $\begin{array}{l}\text { Varianza } \\
\text { explicada } \%\end{array}$ & 4,969 & $\begin{array}{l}\text { Alfa de } \\
\text { Cronbach }\end{array}$ & 0,659 & $\begin{array}{l}\text { Varianza } \\
\text { explicada } \%\end{array}$ & 4,904 & $\begin{array}{l}\text { Alfa de } \\
\text { Cronbach }\end{array}$ & 0,772 \\
\hline \multicolumn{3}{|c|}{ Factor 9: Comunicación y desarrollo } & & \multicolumn{3}{|c|}{$\begin{array}{l}\text { Factor 10: Autonomía y profesiona- } \\
\text { lismo }\end{array}$} & \\
\hline \multicolumn{3}{|c|}{$\begin{array}{l}\text { Existe una buena comunicación entre } \\
\text { la dirección y los trabajadores }\end{array}$} & 0,702 & \multicolumn{3}{|c|}{$\begin{array}{l}\text { Para que un trabajo dé buenos resulta- } \\
\text { dos es necesario que sea hecho con un } \\
\text { alto nivel de conocimientos }\end{array}$} & 0,716 \\
\hline \multicolumn{3}{|c|}{$\begin{array}{l}\text { Todo lo que hay que hacer está claro, } \\
\text { porque se nos explica bien y oportu- } \\
\text { namente }\end{array}$} & 0,635 & \multicolumn{3}{|c|}{$\begin{array}{l}\text { En esta institución los jefes dan las } \\
\text { indicaciones generales de lo que se } \\
\text { debe hacer y se les deja a los emplea- } \\
\text { dos la responsabilidad sobre el trabajo } \\
\text { específico }\end{array}$} & 0,713 \\
\hline \multicolumn{3}{|c|}{$\begin{array}{l}\text { Esta institución se caracteriza por una } \\
\text { preocupación constante acerca del } \\
\text { perfeccionamiento y capacitación del } \\
\text { personal }\end{array}$} & 0,537 & \multirow{2}{*}{\multicolumn{3}{|c|}{$\begin{array}{l}\text { Quienes dirigen esta institución pre- } \\
\text { fieren que uno esté haciendo bien su } \\
\text { trabajo, siga adelante con confianza, en } \\
\text { vez de consultarlo todo con ellos }\end{array}$}} & 0,589 \\
\hline \multicolumn{3}{|c|}{$\begin{array}{l}\text { Existe una buena comunicación entre } \\
\text { los diferentes servicios y unidades que } \\
\text { trabajan generalmente juntos }\end{array}$} & 0,378 & & & & \\
\hline $\begin{array}{l}\text { Varianza } \\
\text { explicada } \%\end{array}$ & 4,707 & $\begin{array}{l}\text { Alfa de } \\
\text { Cronbach }\end{array}$ & 0,707 & $\begin{array}{l}\text { Varianza } \\
\text { explicada } \%\end{array}$ & 4,034 & $\begin{array}{l}\text { Alfa de } \\
\text { Cronbach }\end{array}$ & 0,596 \\
\hline \multicolumn{8}{|c|}{ Factor 11: Excluido } \\
\hline \multicolumn{7}{|c|}{$\begin{array}{l}\text { Aquí se me ha explicado el funcionamiento de la institución, quién tiene la autoridad y } \\
\text { cuáles son las responsabilidades y tareas de cada uno }\end{array}$} & 0,656 \\
\hline \multicolumn{7}{|c|}{ En la medida en que esta institución se desarrolla, siento que yo también me desarrollo } & 0,482 \\
\hline \multicolumn{4}{|c|}{ Varianza explicada\% } & 3,241 & Alfa d & ronbach & 0,389 \\
\hline
\end{tabular}

Elaboración propia a través de spss 15.0 
y la adecuación de los funcionarios a sus respectivas unidades de trabajo. Un elemento clave en este factor, es la confianza que las jefaturas promuevan en la relación con sus "subordinados", para que actúen con profesionalismo, ejerzan autonomía y co-contribuyan al éxito de la organización como un todo, lo cual aprecian y valoran como señal de confianza y respeto de sus desempeños para el logro de las metas institucionales del sector salud (4).

\section{Conclusiones}

Se logró diseñar un cCov en el contexto de dos hospitales de alta complejidad y con una muestra de 561 funcionarios, determinándose 10 factores, con un total de 44 componentes principales activos, equivalentes al $61,97 \%$ de las 71 variables analizadas originalmente, logrando explicar un 52,181\% de la varianza total con un alfa de Cronbach de $0,89 \%$, que ratifica la coherencia y la confiabilidad del instrumento diseñado.

La propuesta de cuestionario para medir el clima organizacional en hospitales de alta complejidad que aquí se valida, se compara positivamente con estudios similares realizados el año 2010 en el ámbito laboral, como es el caso del cuestionario ICO (42) que postula un inventario del clima organizacional, previsto como herramienta para evaluar la calidad del trabajo. De la misma manera, se compara positivamente con la propuesta IPAO (43), del año 2004, con el diseño, construcción y validación de un instrumento que evalúa clima organizacional en empresas colombianas, utilizando la teoría de respuesta al ítem; en ambos casos, se presentan los medios de prueba pertinentes a los hallazgos.

También respecto de la confiabilidad, la propuesta de cuestionario CCOV que se hace en este trabajo, se compara muy bien con el cuestionario sobre clima organizacional universitario del año 2008, C-CLIOU (55), que mide cualidades psicométricas, y con el CFK Ltd. (56) del año 2011 que propone una caracterización psicométrica de un instrumento de clima organizacional en el sector educativo universitario colombiano, donde se aplicó alfa de Cronbach y se alcanzó un índice muy alto de 0,90 .

En otros estudios, sin embargo, que si bien lograron coeficientes elevados mediante un instrumento con formato dicotómico orientado al trabajo profesional y aplicando estructura factorial, fiabilidad y validez del cuestionario de satisfacción S21/26 (48), algunas de sus dimensiones no alcanzaron coeficientes suficientes de validación, como es el caso también de la propuesta del MDCO (57), que postuló el diseño y validación de un modelo de medición del clima organizacional basado en percepciones y expectativas; en un caso y en otro se obtuvieron resultados inferiores al rango de bueno, los cuales fueron calificados de inaceptables $(41,46,49)$.

Es por ello que la propuesta de cuestionario CCOv que aquí se postula, dados sus coeficientes de validación y de confiabilidad, contribuye a generar un instrumento de medición del clima organizacional adaptado a la realidad de hospitales de alta complejidad (58).

Del análisis de factores de componentes principales del CCOV se ratifica el impacto que a priori pueden tener los estudios de clima organizacional, en principio porque el éxito en la gestión del clima organizacional está precisamente en el nivel directivo y se expresa a través del apoyo sistemático a los subordinados; por una parte, coincidiendo con los estudios de la motivación hospitalaria realizados en el contexto chileno (15), porque los personales del sector de la salud valoran a sus jefaturas cuando se les respetan las opiniones y, por otra, cuando desde la 
gestión directiva superior se abren espacios de desarrollo profesional, se ejecutan buenas prácticas y los funcionarios son reconocidos en la comunidad laboral.

Quedan, sin embargo, dentro de las preocupaciones no resueltas algunas cuestiones relevantes, como lo es la percepción negativa o deficitaria de la variable disponibilidad relativa de personas y recursos materiales, la cual según los entrevistados no garantiza que las instituciones el Estado ofrezcan, como gestores de los recursos públicos, los medios suficientes para sus operaciones.

De la misma forma, también como una cuestión deficitaria, se reconoce que los estudios de clima organizacional tienden a ser particularmente parcelados; por una parte, porque recogen solo la percepción de los trabajadores, en algunos casos sobre los temas de la motivación, y porque en otros casos el tema de foco de los estudios es la gestión de la cultura de las organizaciones, que de alguna manera recae en la responsabilidad de los mandos directivos, quienes no siempre la ubican en la primera posición de sus decisiones, generándose de esta forma limitaciones macroambientales de compleja medición a través de estudios de clima.

De manera complementaria, es de reconocer que en este trabajo solo fue posible indagar las percepciones de una muestra de 561 funcionarios; puede ser necesario y valioso realizar estudios de censo a la totalidad de los funcionarios y trabajadores de las instituciones hospitalarias.

A modo de reflexión, los diez factores determinados y los 44 reactivos definidos para medir el clima morganizacional en dos instituciones hospitalarias de alta complejidad, muestran la validez de las variables humanas, de relacionamiento, de confianza y lealtad que dan forma al constructo, de manera que sería necesario y recomendable, a nivel institucional del sector de la salud, realizar investigaciones de similar envergadura y complejidad técnica en distintas reparticiones del sector de la salud, eventualmente extendiendo la aplicación del CCOV al sector de la atención primaria de salud APS; además, de ser factible, su aplicación a entidades hospitalarias de otras regiones de Chile.

Finalmente, y a modo de última proyección de futuras líneas de investigación derivadas de este trabajo, sería muy interesante confirmar la validez de los 10 factores intangibles identificados y sus respectivos 44 componentes del clima organizacional, realizando estudios de envergadura nacional que permitan formalizar los estudios de clima a nivel de todo Chile.

\section{Referencias bibliográficas}

1. Likert R. Una técnica para medir actitudes. Editorial Trillas, 1976. p. 182-93.

2. Halpin A, Crofts D. The organizational climate of schools. International Review of Education. 1963; 22 (4): 441-63.

3. Litwin G, Stringer R. Motivation and organizational climate. Boston: Harvard University Press; 1968.

4. Santa Eulalia JM, Sampedro B. Clima organizacional en instituciones de atención primaria de salud. Rev Med Electrón [serie en Internet]. 2012 [acceso: 2 de noviembre del 2013]; 34 (5): 606-19. Disponible en: http://scielo.sld.cu/scielo.php?script $=$ sci arttext $\&$ pid $=$ S1684-18242012000500011\&lng $=\overline{\mathrm{e}}$ $\mathrm{n} \& \mathrm{nrm}=$ iso.

5. Silva R, de la Torre J, López A, Bastos S. El clima organizacional en el diseño del Balanced Scorecard: Evaluación psicométrica de un instrumento de medida. Revista Contabilidade Vista \& Revista. 2011 Ene-Mar; 22 (1): 107-41.

6. Pérez de Maldonado I, Maldonado M, Bustamante S. Clima organizacional y gerencia: inductores del cambio organizacional. Investigación y Postgrado [serie en Internet]. 2006 [acceso: 13 de enero del 2014]; 21 (2): 231-48. Disponible en: http://www.scielo.org.ve/scielo.php?pid=S131600872006000200009\&script $=$ sci_arttext.

7. Segredo-Pérez AM, Miranda-Reyes D. Clima organizacional en salud pública. Consideraciones generales. Cuba: Escuela Nacional de Salud Pública de Cuba; 2004.

8. Ivancevich JM, Lyon HL. Organizational climate, job satisfaction, role clarity and selected emotional reaction variables in a hospital milieu. Lexington, 
Kentucky: Office of Development Services, University of Kentucky; 1972.

9. Noguera JR, Samudio M. Diagnóstico del clima organizacional del Hospital Central de las Fuerzas Armadas de Paraguay. Mem Inst Investig Cienc Salud. 2014; 12 (1): 14-25.

10. Bernal I, Pedraza N, Sánchez M. El clima organizacional y su relación con la calidad de los servicios públicos de salud: diseño de un modelo teórico. Revista Estudios Gerenciales. 2015 Ene-Mar; 31(134): 8-19.

11. Segredo A, García A, López P, Cabrera P, Perdomo I. Enfoque sistémico del clima organizacional y su aplicación en salud pública. Rev Cub Salud Pública. 2015 Ene-Mar; 41 (1): 115-29.

12. Neal A, Griffin M, Hart P. The impact of organizational climate on safety climate and individual behavior. Safety Science. 2000; 34: 99-109.

13. Segredo A, Reyes D. Clima organizacional en salud pública. Consideraciones generales. Revista Correo Científico Médico de Holguín [serie en Internet]. 2004 [acceso: 12 de julio del 2013]; 8 (3). Disponible en: http://www.cocmed.sld.cu/no83/n83rev4.htm.

14. Vega D, Arévalo A, Sandoval J, Aguilar M, Giraldo J. Panorama sobre los estudios de clima organizacional en Bogotá, Colombia. Revista Diversitas Perspectivas en Psicología. 2006; 2 (2): 329-49.

15. Bustamante MA, del Río MC, Lobos GE, Villarreal PI. Percepción de la motivación de directivos intermedios en tres hospitales complejos de la Región del Maule, Chile. Salud Pública Mex. 2009 Sep.-Oct.; 51(5): 417-26.

16. Carmona V, Jaramillo E. Estudio del clima organizacional en la E.S.E. Hospital San Jorge de Pereira [tesis para obtención de grado]. Colombia: Universidad Tecnológica de Pereira; 2010.

17. Pelaes O. Relación entre el clima organizacional y la satisfacción del cliente en una empresa de servicios telefónicos [tesis para obtención de grado]. Perú: Universidad Nacional Mayor de San Marcos; 2010.

18. Salinas C, Laguna J, Mendoza M. La satisfacción laboral y su papel en la evaluación de la calidad de la atención médica. Salud Pública Mex. 1994 Ene-Feb; 36 (1): 22-9.

19. Hernández V, Quintana L, Guedes R, Mederos R, Sablón N. Estudio de la motivación, la satisfacción, la cultura organizacional y la calidad del servicio existente en el Hospital Mario Muñoz Monroy. Folletos Gerenciales. 2006; 10 (10): 37-57.

20. Delgado A, Bellón J, Martínez M, Luna J, López L, Lardelli P. Las dimensiones del clima organizacional percibidas por los médicos de familia. Aten Primaria. 2006; 37 (9): 489-97.

21. Bermejo M, Bermejo G, de la Rosa G, Montaudon BG. Evaluación del clima organizacional en una unidad hospitalaria de tercer nivel. Rev Cir (Mex). 2008; 75 (1) 50-57.

22. Aldana O, Hernández M, Aguirre D, Hernández S. Clima organizacional en una unidad de segundo nivel de atención. Revista de Enfermería del Instituto Mexicano del Seguro Social. 2009; 17 (2): 91-96.

23. Greenslade J, Jimmieson N. Organizational factors impacting on patient satisfaction: A cross sectional examination of service climate and linkages to nurses' effort and performance. Int J NursStud. 2011 May; 48 (10): 1188-98.

24. Puch M, Ruiz D, Almeida C. Diagnóstico del clima organizacional del Hospital de la Mujer Zacatecana. Revista Salud Pública y Nutrición. 2012; 13 (4): 1-8.

25. Segura A. Clima organizacional: un modo eficaz para dirigir los servicios de salud. Avances en Enfermería. 2012; 30 (1): 107-13.

26. García A, Moro M, Medina M. Evaluación y dimensiones que definen el clima y la satisfacción laboral en el personal de enfermería. Rev Calid Asist. 2010 Jul-Ago; 25 (4): 207-14.

27. Rodríguez A, Retamal M, Lizana J, Cornejo F. Clima y satisfacción laboral como predictores del desempeño: en una organización estatal chilena. Salud Soc. 2011; 2 (2): 219-34.

28. Segredo A. Clima organizacional en la gestión del cambio para el desarrollo de la organización. Rev Cub Salud Pública. 2013; 39 (2): 383-93.

29. Gavin JF. Organizational climate as a function of personal and organizational variables. Appl Psychol. 1975; 60 (1): 135-9.

30. Campbell JP, Dunnette MD, Lawler EE, Weick KE. Managerial behavior, performance, and effectiveness. New York: McGraw-Hill Book Company; 1970.

31. Jones AP, James LR. Psychological climate: dimensions and relationships of individual and aggregated work environment perceptions. Organizational Behaviour and Human Performance. 1979; 23: 201-50.

32. Pritchard RD, Karasick BW. The effect of organizational climate on managerial job performance and satisfaction. Organizational Behaviour and Human Performance. 1973; 9: 126-46.

33. Newman J. Development of a measure of perceived work environment. AMJ. 1977; 20 (4): 520-34.

34. Steers RM. Organizational effectiveness: a behavioral view. Palo Alto, CA: Goodyear Publishing; 1977.

35. Payne RL, Pheysey DC, Pugh DS. Organization structure, organizational climate and group structure: an explanatory study of their relationship in two British manufacturing companies. Occup Psychol. 1971; 45: 45-56.

36. Lawler EE, Hall DT, Oldham G. Organizational climate: relationship to organizational structure, process, and performance. Organizational Behavior Human Performance. 1974; 11: 139-55.

37. Schneider B, Bartlett J. Individual differences and organizational climate: The research plan and questionnaire development. Pers Psychol. 1968; 21: 323-33.

38. Meyer HH. Achievement motivation and industrial climates. En: Taguiri R, Litwin GH, Barnes L, editors. Organizational climate: explorations of a concept. Boston: Division of Research, Graduate School of Business Administration, Harvard University; 1968. p. 35-65.

39. Forehand GA, Gilmer BV. Environmental variations in studies of organizational climate. Psychol Bull. 1964; 6: 361-82. 
40. Friedlander F, Margulies N. Multiple impacts of organizational climate and individual value systems upon job satisfaction. Pers Psychol. 1969; 22: $171-83$

41. Cardona D, Zambrano R. Revisión de instrumentos de evaluación de clima organizacional. Revista Estudios Gerenciales. 2014 Abr-Jun; 30 (131): 184-9.

42. Rodríguez A, Álvarez A, Sosa I, de Vos P, Bonet M, Van Der Stuyft P. Inventario del clima organizacional como una herramienta necesaria para evaluar la calidad del trabajo. Rev Cubana Hig Epidemiol. 2010; 48 (2): 177-96.

43. Gómez CA. Diseño, construcción y validación de un instrumento que evalúa clima organizacional en empresas colombianas, desde la teoría de respuesta al ítem. Acta Colombiana de Psicología. 2004; 11: 97-113.

44. Patterson M, West M, Shackleton V, Dawson J, Lawthom R, Maitlis S, et al. Validating the organizational climate measure: Links to managerial practices, productivity and innovation. Journal of Organizational Behavior. 2005; 26: 379-408.

45. Hernández R, Fernández C, Baptista P. Metodología de la investigación. México D. F.: McGrawHill; 2006.

46. Eyssautier M. Metodología de la investigación. México D.F.: Editorial Thomson; 2006.

47. Likert R. The human organization: its management and value. New York: McGraw-Hill; 1967.

48. Meliá JL, Pradilla JF, Marti N, Sancemi MD, Oliver A, Tomas JM. Estructura factorial, fiabilidad y validez del cuestionario de satisfacción S21/26: un instrumento con formato dicotómico orientado al trabajo profesional. Revista de Psicología Universitas Tarraconensis. 1990; 12 (1/2): 25-39.

49. Ruiz C. Instrumentos de investigación educativa. Barquisimeto, Venezuela: CIDEG; 1998.

50. Álvarez G. El constructo "clima organizacional". Concepto, teorías, investigaciones y resultados relevantes. Revista Interamericana de Psicología Ocupacional. 1992; 11 (1-2): 225-50.

51. Astudillo J. Cuestionario para medir clima organizacional en instituciones financieras [tesis para obtención de grado]. Santiago: Pontificia Universidad Católica de Chile; 1985.

52. Justiniano O. Cuestionario para medir clima organizacional en instituciones educacionales [tesis para obtención de grado]. Santiago: Pontificia Universidad Católica de Chile; 1984.
53. Ministerio Secretaría General de la Presidencia. Ley $\mathrm{n}^{-}$19.628, sobre protección a la vida privada [Internet]. 1999-2012 [actualizado el 12 de febrero del 2012; acceso: 15 de enero del 2014]. Disponible en: http://www.leychile.cl/ Navegar?idNorma $=141599$.

54. Ministerio de Economía. Ley $N^{\circ} 17.374$, fija nuevo texto refundido, coordinado y actualizado del DFL. $n^{\circ} 313$ de 1960, que aprobara la ley orgánica dirección estadística y censos y crea el Instituto Nacional de Estadísticas [Internet] [actualizado el 29 de julio del 2004; acceso: 15 de enero del 2014]. Disponible en: http://www.leychile.cl/ Navegar?idNorma $=28960$.

55. Mujica M, Pérez I. Cuestionario sobre clima organizacional universitario. Cualidades psicométricas. Educare [Internet]. 2008 [acceso: 21 de septiembre del 2012]; 12(1). Disponible en: http://revistas.upel. edu.ve/index.php/educare/article/view/41/40.

56. Castillo L, Lengua C, Pérez PA. Caracterización psicométrica de un instrumento de clima organizacional en el sector educativo universitario colombiano. Int J Psychol Res (Medellín). 2011; 4 (1): 40-7.

57. Reinoso H, Araneda B. Diseño y validación de un modelo de medición del clima organizacional basado en percepciones y expectativas. Revista Ingeniería Industrial. 2007; 6 (1): 39-54.

58. Ministerio de Salud de Chile. Resolución exenta $\mathrm{n}^{\circ}$ 646: Norma técnica $\mathrm{n}^{\circ} 0150$ sobre criterios de clasificación según nivel de complejidad de establecimientos hospitalarios [Internet]. 2013 [acceso: 7 de abril del 2014]. Disponible en: http://www.deis. cl/wp-content/uploads/2013/08/NT_0150.pdf.

59. Servicio de Salud del Maule Chile: Recursos Online del Servicio de Salud del Maule [Internet]. 2015 [actualizado s/f; acceso: 13 de enero del 2014]. Disponible en: http://www.ssmaule.cl/minsal.

60. DeLorme DE, Huh J, Reid LN. Age differences in how consumers behave following exposure to DTC advertising. Health Commun. 2006 Nov 12; 20 (3): 255-65.

61. García-Bellido R, González J, Jornet JM. spss: Análisis de fiabilidad: Alfa de Cronbach [Internet]. 2010 [actualizado 2010; acceso: 4 de noviembre del 2013]. Disponible en: http://www.uv.es/innomide/ spss/SPSS/SPSS_0801B.pdf. 